\title{
Universiteit
}

Leiden

The Netherlands

\section{Explaining the scatter in the galaxy mass-metallicity relation with gas} flows

Loon, M.L. van; Mitchell, P.D.; Schaye, J.

\section{Citation}

Loon, M. L. van, Mitchell, P. D., \& Schaye, J. (2021). Explaining the scatter in the galaxy massmetallicity relation with gas flows. Monthly Notices Of The Royal Astronomical Society, 504(4), 4817-4828. doi:10.1093/mnras/stab1254

Version: $\quad$ Accepted Manuscript

License: $\quad$ Leiden University Non-exclusive license

Downloaded from: https://hdl.handle.net/1887/3264082

Note: To cite this publication please use the final published version (if applicable). 


\title{
Explaining the scatter in the galaxy mass-metallicity relation with gas flows
}

\author{
Maria L. van Loon ${ }^{\star 1}$, Peter D. Mitchell ${ }^{1}$ and Joop Schaye ${ }^{1}$ \\ ${ }^{1}$ Leiden Observatory, Leiden University, P.O. Box 9513, 2300 RA Leiden, the Netherlands
}

2 January 2022

\begin{abstract}
The physical origin of the scatter in the relation between galaxy stellar mass and the metallicity of the interstellar medium, i.e. the Mass-Metallicity Relation (MZR), reflects the relative importance of key processes in galaxy evolution. The EAGLE cosmological hydrodynamical simulation is used to investigate the correlations between the residuals of the MZR and the residuals of the relations between stellar mass and, respectively, specific inflow, outflow and star formation rate as well as the gas fraction for central galaxies. At low redshift, all these residuals are found to be anti-correlated with the residuals of the MZR for $M_{\star} / \mathrm{M}_{\odot} \lesssim 10^{10}$. The correlations between the residuals of the MZR and the residuals of the other relations with mass are interrelated, but we find that gas fraction, specific inflow rate and specific outflow rate all have at least some independent influence on the scatter of the MZR. We find that, while for $M_{\star} / \mathrm{M}_{\odot}>10^{10.4}$ the specific mass of the nuclear black hole is most important, for $M_{\star} / \mathrm{M}_{\odot} \lesssim 10^{10.3}$ gas fraction and specific inflow rate are the variables that correlate most strongly with the MZR scatter. The timescales involved in the residual correlations and the time that galaxies stay above the MZR are revealed to be a few Gyr. However, most galaxies that are below the MZR at $z=0$ have been below the MZR throughout their lifetimes.
\end{abstract}

Key words: galaxies: formation - galaxies: evolution - metallicity

\section{INTRODUCTION}

The processes involved in galaxy formation and evolution are coupled and take place over a range of length and time scales, which makes it challenging to develop a full understanding. Hydrodynamical simulations are an established tool to assist in this, and modern simulations of cosmological volumes now reproduce many observational constraints well enough (e.g. Vogelsberger et al. 2014, Schaye et al. 2015, Davé et al. 2016, Dubois et al. 2016) that they can be used with confidence to study aspects of galaxy evolution that are not readily observable, such as galactic gas accretion.

Observationally, such processes can be linked indirectly to quantities that are observable, such as the metallicity of the insterstellar medium (ISM). Gas-phase metallicity is influenced by processes that create or deplete heavy elements within galaxies, and by processes that increase or decrease the total gas content of galaxies (see Maiolino \& Mannucci 2019 for a recent review). The metallicity of the ISM is therefore influenced by stellar evolution via the distribution of the metals created inside stars, by galactic outflows that carry metals and gas away from the ISM, and by gas accretion onto the ISM, which dilutes its metal abundance assuming that

\footnotetext{
* E-mail: mvanloon98@gmail.com
}

the surrounding circum-galactic medium (CGM) is comparatively metal poor.

It is well established observationally that galaxy gas-phase metallicity correlates with galaxy stellar mass (e.g. Tremonti et al. 2004, Zahid et al. 2014, Curti et al. 2020), with a scaling relationship that is generally referred to as the Mass-Metallicity Relation (MZR). The MZR has a positive slope that is approximately constant for $M_{\star} / \mathrm{M}_{\odot} \lesssim 10^{10.5}$ and flattens for higher masses. It is usually accepted that the positive slope of the MZR for lower stellar mass galaxies is determined by the higher efficiency of galactic outflows at lower masses, due to their shallower gravitational potentials (e.g. Larson 1974).

The scatter in the MZR is 0.1-0.2 dex, which is more than expected from measurement errors (Maiolino \& Mannucci|2019), and understanding this scatter offers the prospect of advancing in turn our understanding of the underlying network of inflows, outflows, and star formation within galaxies (e.g. Forbes et al. 2014, Finlator \& Davé 2008, Guo et al. 2016, Lara-López et al. 2019, Wang \& Lilly 2020a, De Lucia et al. 2020a). Observationally, it has been reported that metallicity anti-correlates with star formation rate or specific star formation rate at fixed stellar mass (e.g. Ellison et al. 2008, Mannucci et al. 2010, Curti et al. 2020), possibly forming a "fundamental" relation between the three quantities that is invari- 
ant with redshift (Lara-López et al. 2010, Mannucci et al. 2010). Other observational studies do not find a residual correlation between star formation rate and metallicity at fixed stellar mass however (Sánchez et al. 2013 2019). Correlated residuals of the MZR with atomic gas mass or gas fraction (Bothwell et al. 2013) and for molecular hydrogen mass (Bothwell et al. 2016) have also been reported. Generally speaking, theoretical models and cosmological simulations predict qualitatively similar anti-correlations of metallicity with star formation rate or gas mass at fixed stellar mass (e.g. Yates et al. 2012, Lilly et al. 2013; Lagos et al. 2016, De Rossi et al. 2017, Torrey et al. 2019, De Lucia et al. 2020b).

Theoretical work has also been used to study the connection betwen the MZR and variables that are not readily observable. For example, using simple idealised galaxy evolution models, Forbes et al. (2014) and Wang \& Lilly (2020a) study how fluctuations in gas inflows rates can drive the MZR scatter, emphasing the importance of the relationship between the fluctuation timescale for inflows and the gas consumption timescale in the ISM. De Lucia et al. (2020b) use a more complex semi-analytic model of galaxy formation to study the physical drivers of the MZR relation, and arrive at the conclusion that gas accretion drives the MZR scatter, with galaxies below the MZR relation being driven by sustained periods of gaseous inflow. Using hydrodynamical simulations of a cosmological volume, Torrey et al. (2019) arrive at similar conclusions by studying how galaxies evolve in metallicity, gas mass, and star formation rate over finite time intervals.

Here, we extend this body of work by using cosmological hydrodynamical simulations of a representative volume to directly study the connection between the MZR and inflow rates, outflow rates, and star formation rates. Specifically, we use the EAGLE simulations, building on previous analyses of the MZR in these simulations by Schaye et al. (2015), Crain et al. (2015), Lagos et al. (2016), De Rossi et al. (2017), Almeida \& Dalla Vecchia (2018), Trayford \& Schaye (2019) and Zenocratti et al. (2020). Our work complements that of more idealised modelling that generally infers the properties of inflows and outflows based on observables, and also complements semi-analytic modelling by self-consistently allowing for potential coupling between outflows and inflows (i.e. “preventative feedback", e.g. Davé et al. 2012, Mo \& Mao 2002, van de Voort et al. 2011).

This study is organised as follows. Section 2 briefly describes the EAGLE simulations and how the various variables used in this study have been measured and defined. Section 3 presents the results of the study, including some individual examples, and the correlations between the residuals of the MZR and residuals of the relations between stellar mass and the other variables (specific inflow, outflow and star formation rates and the gas fraction). We explore the stellar mass dependence (section 3.1), inter-variable coupling (section 3.2, the contribution to the MZR scatter (section 3.3, and the relevant timescales (section 3.4). Section 4 provides a summary of the main results.

\section{METHODS}

We use data from the EAGLE simulation project, which consists of a suite of cosmological hydrodynamical simulations (Schaye et al. 2015, Crain et al. 2015), which have been made public (McAlpine et al.2016). EAGLE simulates representative cosmological volumes, including gas, stars, supermassive black holes and dark matter, using smoothed particle hydrodynamics (SPH) to solve the equations of hydrodynamics with a modified version of the
GADGET-3 code (last described in Springel 2005, see Schaller et al. 2015 for the employed formulation of SPH.) The simulation assumes a $\Lambda$ CDM cosmological model and uses 'subgrid' models for relevant unresolved physics. The parameters for the subgrid models governing stellar and AGN feedback were calibrated to fit the observed galaxy stellar mass function at $z \approx 0$, as well as the local disk galaxy size - stellar mass relation and the local black hole mass - stellar mass relation.

The EAGLE simulation suite consists of a set of "Reference" simulations with a common set of model parameters, as well as a number of variations. All EAGLE measurements in this article are taken from the largest Reference model simulation, RefL100N1504, which simulates a $(100 \mathrm{Mpc})^{3}$ box volume with $1504^{3}$ dark matter and $1504^{3}$ baryonic particles. The (initial) particle masses in this simulation are $1.8 \times 10^{6} \mathrm{M}_{\odot}$ for gas and stars, and $9.7 \times 10^{6} \mathrm{M}_{\odot}$ for dark matter.

Since EAGLE employs SPH, it is possible to track simulation particles across time. We use this information to measure galactic inflow and outflow rates by determining how much gas enters or leaves the ISM of a given galaxy between two simulation snapshots. The precise method used to measure inflow and outflow rates is described in detail in Mitchell et al. (2020a. p). We define the ISM as the combination of star-forming gas (meaning its density exceeds the metallicity-dependent threshold introduced in Schaye 2004), and also non-star forming gas with density $n_{\mathrm{H}}>0.01 \mathrm{~cm}^{-3}$ that is located within $20 \%$ of the halo virial radius, approximately matching a selection of neutral atomic hydrogen (e.g. Rahmati et al. 2013).

To account for particles fluctuating across the ISM boundary, outflowing gas particles are selected by their radial velocity, ensuring that they have moved a significant distance. The instantaneous radial velocity and the difference in radius between two subsequent snapshots, divided by the time elapsed between them, must both exceed 0.25 times the maximum circular velocity of the halo. Particles that leave the ISM without meeting these criteria are tracked through subsequent snapshots until they: $a$ ) meet the outflow criteria, or $b$ ) have re-entered the ISM (at which point they are not counted towards the inflow rate), or $c$ ) until three halo dynamical times have passed.

Inflowing gas particles can be distinguished into three different types, as presented by Mitchell et al.(2020a). First, particles can be accreted onto a galaxy for the first time. Second, particles can be recycled from a progenitor of the current galaxy. Last, particles can be transferred from the ISM of another galaxy. These categories are added together to create the total inflow onto a galaxy, which is the variable used in this study. Note that a distinction is made between inflows and gas accretion due to galaxy mergers; we choose not to include the latter in our analysis (defining mergers as satellites with a maximum past halo mass greater than $9.7 \times 10^{8} \mathrm{M}_{\odot}$, corresponding to 100 dark matter particles at fiducial EAGLE resolution).

Inflow, outflow, and star formation rates are measured using adjacent snapshots in a 200 snapshot grid. Inflowing (outflowing) gas mass, or formed stellar mass between snapshots is divided by the time elapsed between snapshots, which is $\approx 120 \mathrm{Myr}$ at low redshift and becomes smaller at higher redshifts (for the exact temporal spacing, see figure A1 in Mitchell et al. 2020b). In parts of our analysis, the temporal spacing is adjusted by tracking galaxies across snapshots and integrating their inflow, outflow or star formation rates over longer timescales, which we fix to be a constant fraction of the halo dynamical time.

This study focusses only on central galaxies, thereby simplifying the interpretation of the results by excluding environmen- 


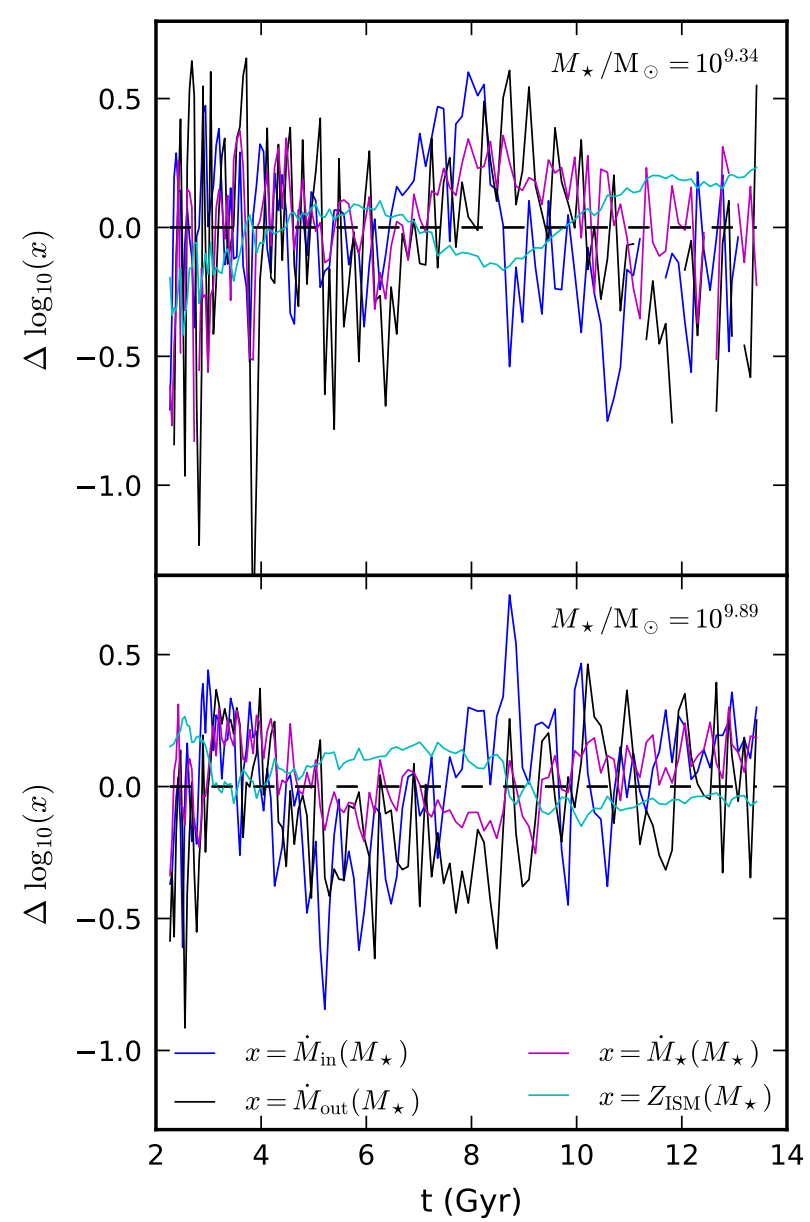

Figure 1. Examples of the residual inflow rate $\left(\dot{M}_{\text {in }}\right)$, outflow rate $\left(\dot{M}_{\text {out }}\right)$, star formation rate $\left(\dot{M}_{\star}\right)$ and metallicity of the interstellar medium $\left(Z_{\mathrm{ISM}}\right)$ of two individual galaxies tracked through time, with present-day masses $M_{\star} / \mathrm{M}_{\odot}=10^{9.34}$ (top) and $M_{\star} / \mathrm{M}_{\odot}=10^{9.89}$ (bottom). At $z=0$, samples of galaxies are chosen based on stellar mass (Top: $M_{\star} / \mathrm{M}_{\odot}=$ $10^{9.4 \pm 0.3}$; Bottom: $M_{\star} / \mathrm{M}_{\odot}=10^{10.0 \pm 0.3}$ ). Residuals are taken with respect to the medians for these same samples at each snapshot. The inflow, outflow and star formation rate show fluctuations across a wide range of timescales. Metallicity anti-correlates with these variations but it fluctuates less strongly.

tal effects. Furthermore, galaxies with zero ISM gas particles are excluded due to their undefined metallicity. Similarly, for figures showing data concerning inflows, galaxies with zero inflow rate have been excluded (the same holds for outflows and star formation rate (SFR)). Apart from this selection, we select galaxies only by stellar mass and redshift. However, most figures show (with transparent and dashed lines) where the interpretation becomes uncertain due to a majority of galaxies $(>50 \%)$ containing $<20$ inflowing, outflowing, newly formed star particles, or $<200$ star forming gas particles. Furthermore, we discard data points including fewer than 10 galaxies, or where $>50 \%$ of galaxies have $<10$ inflowing, outflowing, newly formed star particles, or $<100$ star forming gas particles, to reduce the noise in the plots. None of these choices strongly influence the results.

\section{RESULTS}

Fig. 1] shows two examples of typical star forming galaxies, whose inflow rate, outflow rate, star formation rate and metallicity have been tracked though time. At $z=0$ samples of the galaxies are created based on their stellar mass (bin width $=0.3$ dex). These samples are then used to determine the median values in each snapshot, with respect to which the residuals of the variables are calculated. Fig. 1 plots the evolution of there residuals for two individual galaxies. It illustrates clearly that when the inflow, outflow and star formation rates are higher than the median of the sample (i.e. the residual is positive), the metallicity tends to be lower (i.e. the residual is negative) and vice versa. The variables fluctuate over a range of timescales spanning $\approx 0.25 \mathrm{Gyr}$ to $\approx 5 \mathrm{Gyr}$, where the longer timescale fluctuations show a clear anti-correlation between metallicity and the other variables. Inflow rates (blue line) generally seem to be the first to rise or fall, after which other variables react, if these (anti-) correlations are interpreted causally. The short timescale fluctuations of inflow, outflow and star formation rate are large compared to those in the metallicity.

Fig. 2 shows the median residuals of the relations between stellar mass and, respectively, the specific inflow rate, specific outflow rate, gas fraction and specific star formation rate plotted against the residuals of the mass-metallicity relation for different stellar mass bins $\left(M_{\star} / \mathrm{M}_{\odot}=10^{9.0 \pm 0.3}\right.$ (blue), $M_{\star} / \mathrm{M}_{\odot}=$ $10^{10.0 \pm 0.3}$ (green), $M_{\star} / \mathrm{M}_{\odot}=10^{11.0 \pm 0.3}$ (magenta)), at $z<0.1$. The 'specific' in specific inflow/outflow/star formation rate means the variables are divided by the stellar mass of the galaxy, thus factoring out any residual stellar mass dependence remaining within the finite stellar mass bins.

Focusing first on the stellar mass bin $M_{\star} / \mathrm{M}_{\odot}=10^{10.0 \pm 0.3}$, the residual specific inflow rate, specific outflow rate, gas fraction and specific star formation rate all anti-correlate with the residual metallicity (see Fig. 2. green lines in the top left, top right, bottom left, bottom right panels, respectively). When interpreted causally, this suggests that the processes responsible for increasing these variables generally lower the gas metallicity in a galaxy, with a possible lower limit to the effect (the correlations flatten at extremely low values). Due to the coupling of the variables however (see sections 1 and 3.2), the correlations need to be investigated further before understanding which physical processes cause them.

A starting point for uncovering the physical origin of the MZR scatter can be a comparison between the correlations shown in Fig. 2. A comparison of slopes reveals that the correlations of the residual metallicity with the residual specific inflow and outflow rate match closely in steepness and shape, whereas the residual correlations with the gas fraction and sSFR residual correlations are much steeper, the gas fraction residual correlation being the steepest. This supports the idea that the correlation with the gas fraction may be caused by a combination of the influence of multiple other variables.

\subsection{Stellar mass dependence}

Fig. 2 depicts the residual correlations for three stellar mass bins, of $M_{\star} / \mathrm{M}_{\odot}=10^{9.0 \pm 0.3}, 10^{10.0 \pm 0.3}$ and $10^{11.0 \pm 0.3}$, at $z<0.1$. The redshift evolution of the residual correlations has also been investigated and is found to be small, as can be viewed in Appendix A Considering the stellar mass dependence of the residual correlations, Fig. 2 shows that the lower $\left(M_{\star} / \mathrm{M}_{\odot}=10^{9.0 \pm 0.3}\right.$, blue lines in Fig. 2 and intermediate $\left(M_{\star} / \mathrm{M}_{\odot}=10^{10.0 \pm 0.3}\right.$, green lines in 

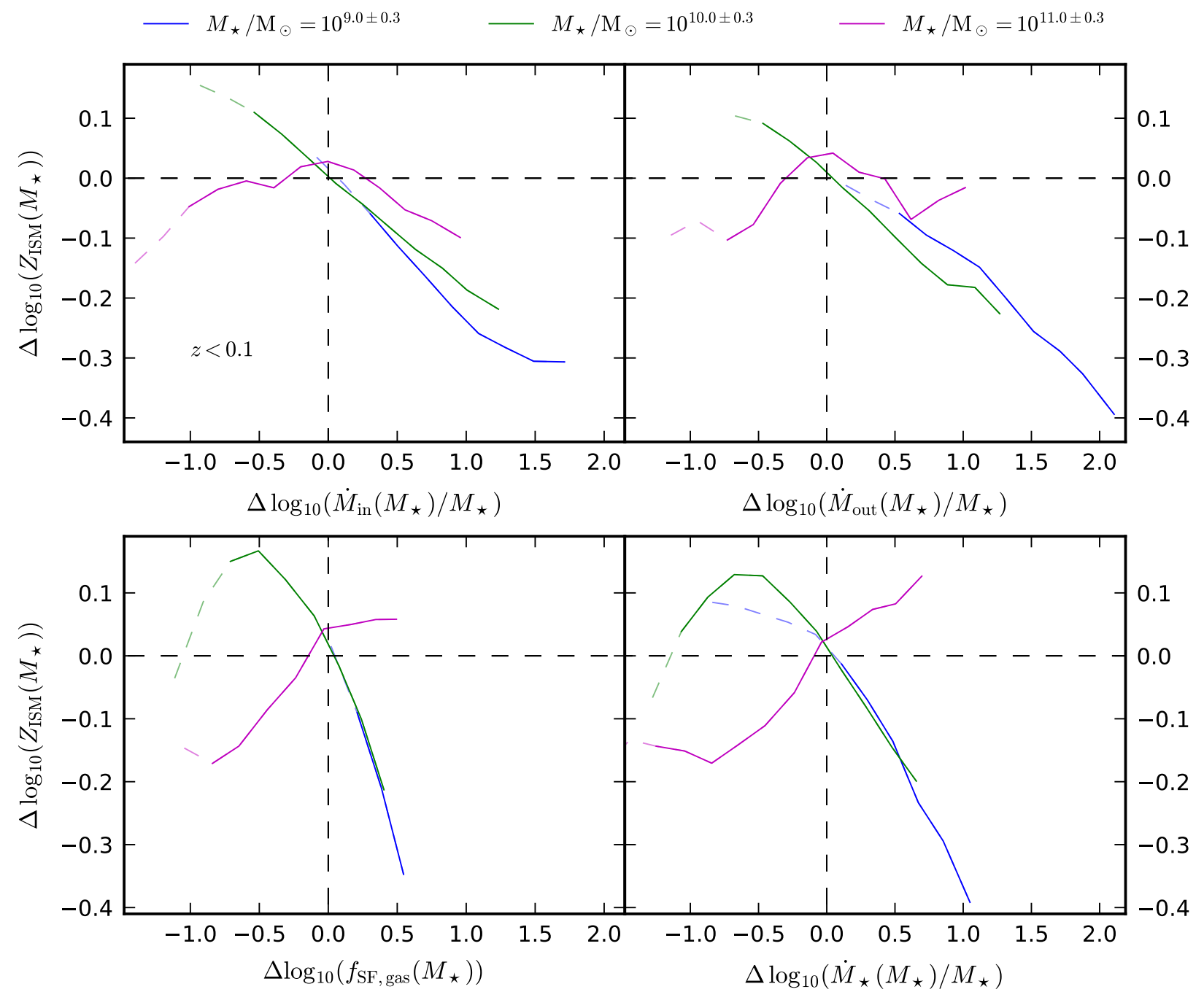

Figure 2. The relations between the median residual metallicity of the interstellar medium and residual specific inflow rate (top left), residual specific outflow rate (top right), residual gas fraction (bottom left) and residual sSFR (bottom right) for central galaxies with $M_{\star} / \mathrm{M}_{\odot}=10^{9.0 \pm 0.3}$ (blue lines), $M_{\star} / \mathrm{M}_{\odot}=$ $10^{10.0 \pm 0.3}$ (green lines), $M_{\star} / \mathrm{M}_{\odot}=10^{11.0 \pm 0.3}$ (magenta lines), at $z<0.1$. The residuals are taken w.r.t. the medians as a function of stellar mass. A dashed and transparent line style indicates regions where more than $50 \%$ of galaxies in the bin contain $<20$ inflowing (top left panel), outflowing (top right panel), newly formed star particles (bottom right panel) or $<200$ star forming gas particles (bottom left panel). The lines are cut off when more than $50 \%$ of galaxies in the bin contain $<10$ inflowing (top left panel), outflowing (top right panel), newly formed star particles (bottom right panel), or $<100$ star forming gas particles (bottom left panel). or when bins contain fewer than 10 galaxies. For stellar masses $<10^{10.3} \mathrm{M}_{\odot}$, the shapes and slopes are comparable across the residual correlations. For stellar masses $>10^{10.7} \mathrm{M}_{\odot}$, they deviate, forming two groups: specific inflow and outflow rate (top), and gas fraction and sSFR (bottom).

Fig. 2) stellar mass bins of all four residual correlations show a comparable progression, varying only slightly in shape and slope.

The higher stellar mass bin $\left(M_{\star} / \mathrm{M}_{\odot}=10^{11.0 \pm 0.3}\right)$ deviates in shape from the lower two, especially for the residual trends with gas fraction and star formation rate, which change in sign to clear positive correlations, consistent with the previous analysis of EAGLE by De Rossi et al. (2017) (see also Yates et al. 2012 for a similar result from a semi-analytic model). The residual correlations with inflow and outflow rate deviate in a more complex manner, appearing to show a positive correlation for negative $\mathrm{x}$-axis residuals, but retaining an overall anti-correlation for positive $\mathrm{x}$-axis residuals.

By comparing EAGLE simulations with and without AGN feedback, De Rossi et al. (2017) find that the inversion of the dependence on the gas fraction and star formation rate residuals at high stellar mass can be attributed to the AGN feedback. We explore this aspect further in Fig. 3, which shows the correlation between residual ISM metallicity and residual specific black hole mass. Fig. 3 supports the idea that for $M_{\star} / \mathrm{M}_{\odot}>10^{10.7}$ the shapes of the residual correlations (Fig. 2) are likely caused by the influence of AGN feedback on larger mass galaxies. For the highest mass bin, the correlation between residual metallicity and residual specific black hole mass is steeper and negative, suggesting that high black hole masses correlate strongly with a low metallicity. For the lower stellar mass bins, the residual correlations are almost flat until extreme positive residuals in black hole mass, indicating a possible 


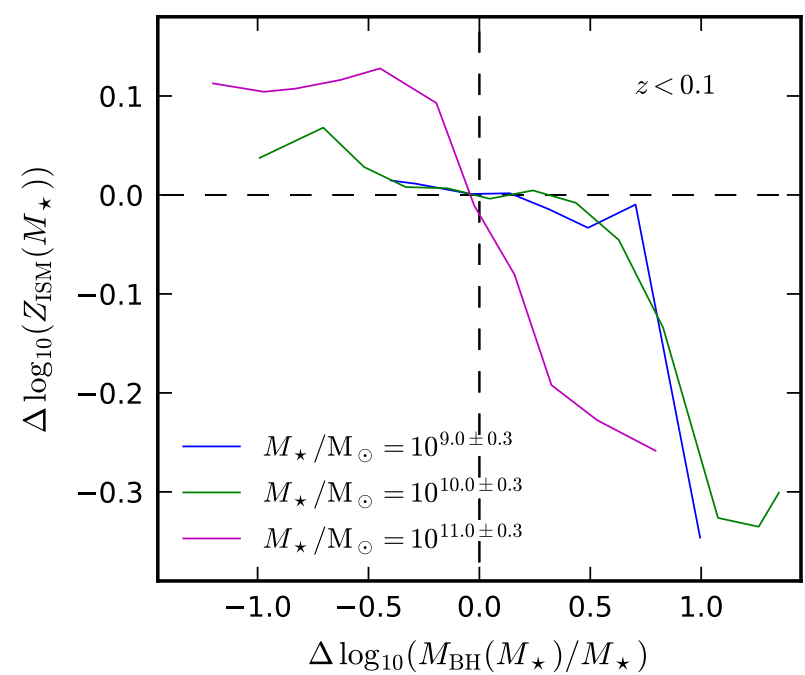

Figure 3. The median residual of the relation between ISM metallicity and stellar mass as a function of the residual between specific black hole mass and stellar mass for central galaxies with $M_{\star} / \mathrm{M}_{\odot}=10^{9.0 \pm 0.3}$ (blue line), $M_{\star} / \mathrm{M}_{\odot}=10^{10.0 \pm 0.3}$ (green line), $M_{\star} / \mathrm{M}_{\odot}=10^{11.0 \pm 0.3}$ (magenta line), at $z<0.1$. Only bins containing more than 10 galaxies are shown. The anti-correlation between residual metallicity and residual specific black hole mass is only significant for relatively high black hole masses and is steeper for the highest mass bin.

causal connection whereby black holes above a threshold mass are able to influence the metallicity of the galaxy.

\subsection{Coupling between different variables}

While Fig. 2 shows clear correlations between metallicity and inflow, outflow, and star formation rates, and also gas fraction, the latter four variables can of course also be correlated with each other, rather than each directly driving a causal connection. One way to probe the coupling of variables, is by correcting the residual correlations for others, thus making it possible to judge the influence of each variable on the other residual correlations. By comparing all possibilities, insight is gained into which variable may be most fundamental. In Fig. 4. each panel shows the residual correlation of one of the four variables (black lines, $M_{\star} / \mathrm{M}_{\odot}=10^{10.0 \pm 0.3}, z<0.1$; there curves are identical to the green lines in Fig. 2, and the residual correlation after correcting for one of the other variables (blue: specific inflow rate, green: specific outflow rate, magenta: specific star formation rate, cyan: gas fraction).

The correction is calculated by taking the residuals of the residual correlations. First, the median residuals of the relations between stellar mass and, respectively, metallicity and (e.g.) specific outflow rate are calculated (respectively $\Delta \log _{10}\left(Z_{\operatorname{ISM}}\left(M_{\star}\right)\right)$ and $\Delta \log _{10}\left(\dot{M}_{\text {out }}\left(M_{\star}\right) / M_{\star}\right)$, which are plotted in Fig. 2$)$. Then, the residuals of the relation between these two variables are calculated, creating a new variable:

$$
\Delta\left(\Delta \log _{10}\left(Z_{\mathrm{ISM}}\left(M_{\star}\right)\right)\left[\Delta \log _{10}\left(\dot{M}_{\text {out }}\left(M_{\star}\right) / M_{\star}\right)\right]\right)
$$

which is then plotted against the residuals of the relation between stellar mass and another variable (e.g. specific inflow rate) to create Fig. 4.

Fig. 4 shows that each residual relation flattens considerably after correcting for another residual correlation (compare the coloured lines to the black lines), indicating that none of the variables are fully independent. When studying the bottom right panel in Fig. 4. it becomes clear that the effect of the sSFR on the residual metallicity is completely determined by the gas fraction (cyan line) and to a lesser extent by the specific inflow and outflow rates (blue and green lines, respectively), with the exception of extreme values $\left(\Delta \log _{10}(\mathrm{sSFR})<-0.7, \Delta \log _{10}(\mathrm{sSFR})>0.3\right)$. The relations between residual metallicity and, respectively, residual specific inflow rate, residual specific outflow rate and residual gas fraction flatten considerably when corrected for the other variables. However, there is no significant difference between them.

Combining all the information in Fig. 4 we can conclude that the gas fraction, specific inflow, outflow and star formation rate all play an independent role in determining the metallicity of a galaxy, but that they are also coupled. The independent influence of star formation rate is least important. The gas fraction can be seen physically as a time integrated aggregate of the three other processes, and will therefore naturally correlate strongly with the metallicity and affect other correlations.

\subsection{The scatter in the MZR}

We now consider directly how much of the MZR scatter can be explained by each of the variables considered, using a similar method as used by Matthee \& Schaye (2019) to explore the origin of the scatter in star formation rates at fixed stellar mass in the EAGLE simulation. Fig. 5 shows how much of the original MZR scatter (black line) remains after correcting for the residual correlation with another variable, using the procedure described in section 3.2 Fig. 5 then depicts the remaining $(1 \sigma)$ scatter. The residual correlations were recalculated for different stellar mass bins (width $=0.15$ dex) to account for the stellar mass dependencies of the residual correlations and include only central galaxies at $z<0.1$.

Fig. 5 shows that up to $\approx 0.06$ dex of the $\approx 0.13$ dex scatter in the MZR can be explained by correcting for one of the variables (e.g. the gas fraction, cyan line). At low stellar masses $\left(M_{\star} / \mathrm{M}_{\odot}<\right.$ $10^{10.4}$ ) correcting for the gas fraction (cyan line) or the specific inflow rate (blue line) reduces the scatter the most, marking these two variables as most influential. When correcting for the SSFR (magenta line) or specific outflow rate (green line) residual correlation, only $\approx 0.025$ dex in scatter is explained at $M_{\star} / \mathrm{M}_{\odot}<10^{9.3}$, compared to the $\approx 0.04$ dex at $M_{\star} / \mathrm{M}_{\odot} \approx 10^{9.6}$. At $M_{\star} / \mathrm{M}_{\odot}>10^{10}$ the amount of explained scatter diminishes for all variables, with the exception of specific black hole mass (yellow line), which only accounts for scatter at higher stellar masses and is the most important variable for $M_{\star} / \mathrm{M}_{\odot}>10^{10.4}$. This can be explained by the effects of AGN feedback on the metallicity of high-mass galaxies.

Fig.5 provides strong evidence supporting the idea of inflows of gas having a larger influence on the scatter in the MZR than outflows or star formation for $M_{\star} / \mathrm{M}_{\odot} \lesssim 10^{10}$. The gas fraction reduces the scatter in the MZR the most, which supports the idea that the physical origin of the scatter lies in more than one variable, under the assumption that the gas fraction reflects the influence of multiple variables (inflow, outflow and star formation rates). Note that this does not exclude the possibility of a direct influence of the gas fraction on the metallicity (higher gas surface densities could change the effectiveness of feedback, for example).

\subsection{Timescales}

Fig. 5 shows that not all of the scatter in the MZR can be explained by just one of the individual variables considered. We remind the 

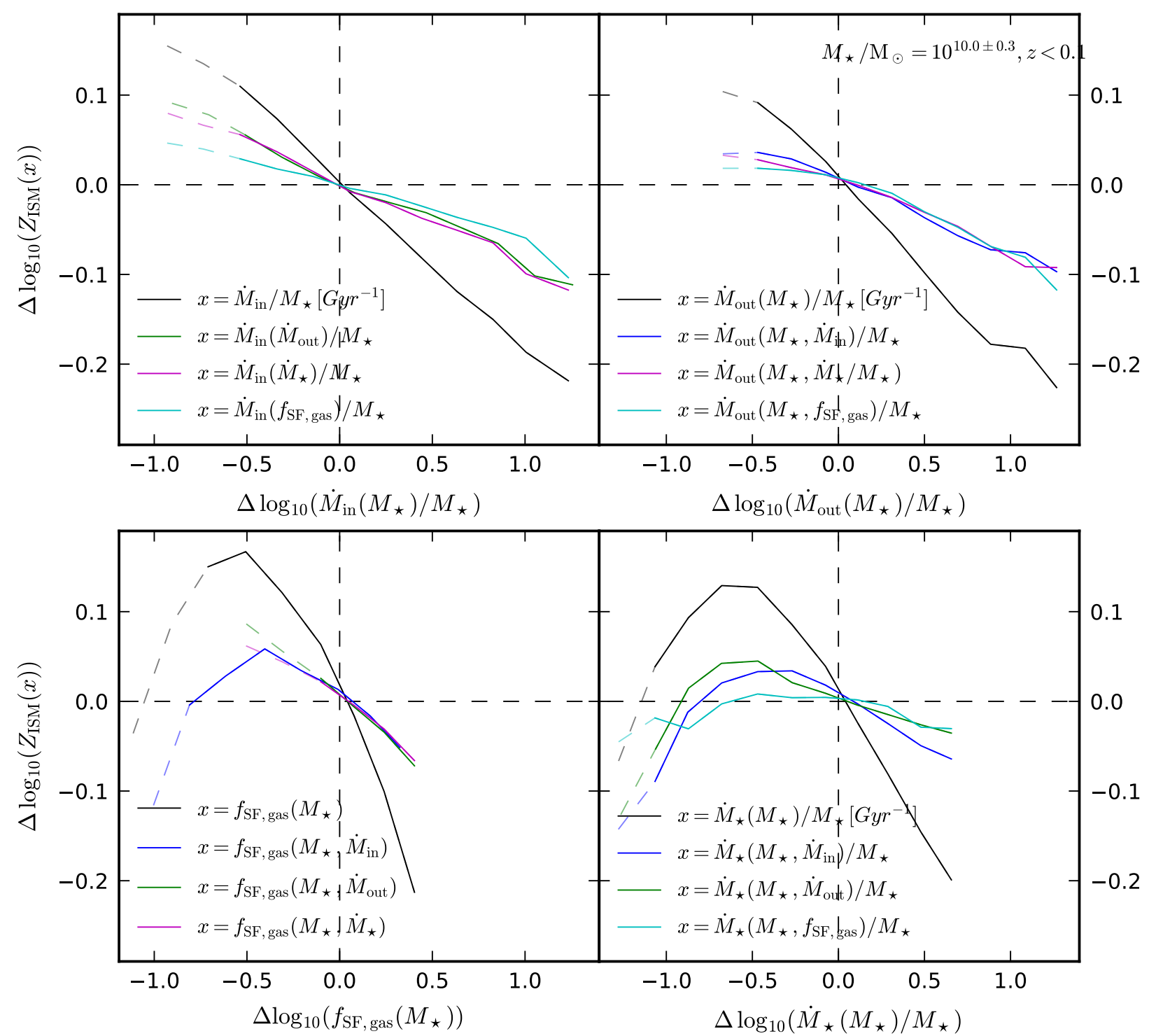

Figure 4. The median residual of the gas mass-metallicity relation as a function of the residual specific inflow rate (top left), specific outflow rate (top right), gas fraction (top left), and sSFR (bottom right) for central galaxies with $M_{\star} / \mathrm{M}_{\odot}=10^{10.0 \pm 0.3}$, at $z<0.1$. Black: The residuals of the relations with stellar mass. Blue: The residual correlation corrected for the relation between residual metallicity and residual specific inflow rate. Green: The residual correlation corrected for the relation between residual metallicity and residual specific outflow rate. Magenta: The residual correlation corrected for the relation between residual metallicity and residual specific star formation rate. Cyan: The residual correlation corrected for the relation between residual metallicity and residual the gas fraction. Line styles are as in Fig.2. Each residual correlation flattens significantly when corrected for the dependence of metallicity on one of the other variables implying that all variables are coupled.

reader however that until now we have presented inflow and outflow rates that are measured over $\mathrm{a} \approx 120 \mathrm{Myr}$ time interval. Recalling from Fig. 1 that the metallicity shows long timescale $(\gg 1$ Gyr) fluctuations, we now investigate the timescales involved in the residual correlations by averaging the variables over longer time intervals.

Fig. 6 illustrates that the correlations with the residual specific inflow, outflow and star formation rate are likely dominated by fluctuations on long timescales. The figure shows the residual correlations for measurements of the variables over different time intervals, which smooth the fluctuations that occur on shorter timescales. Galaxies selected at $z<0.1$ are tracked back in time, across the desired time interval, and their inflows/outflows/formed stars are added together.

When the time interval increases, smoothing the shorter timescale fluctuations, the relations with the residual specific inflow and outflow rates steepen somewhat (top panels). This slight increase of steepness is due to the decrease of 'noise' (shorter timescale fluctuations in the variables), indicating that fluctuations on longer timescales are likely the main cause of the correlations. The residual correlation for specific outflow rate saturates at a time interval of $>1 \mathrm{Gyr}$, suggesting that fluctuations on this timescale can no longer be considered to be noise. The inflow and outflow panels indicate that short timescales are not dominating the resid- 


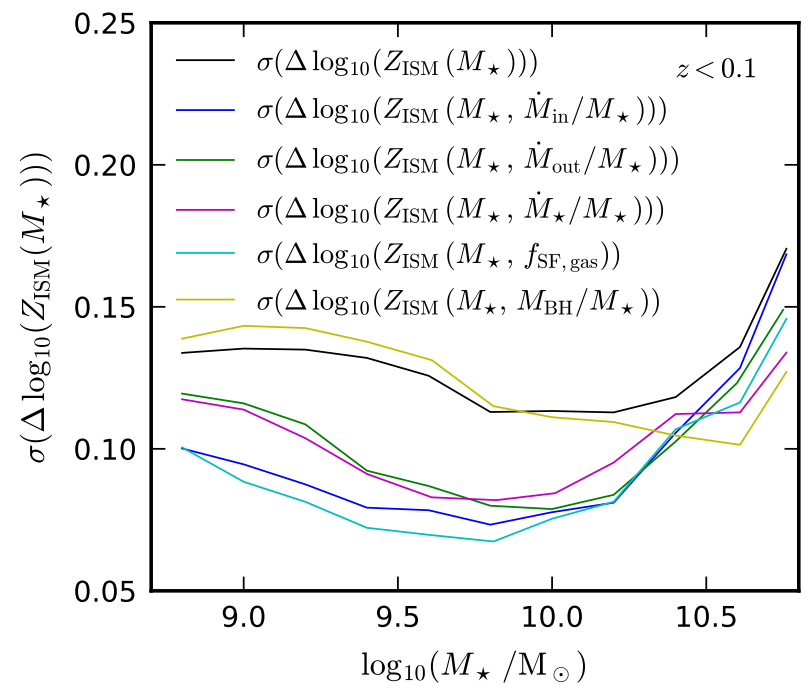

Figure 5. The mass dependence of the $(1 \sigma)$ scatter in the median MZR (black), when corrected for the correlations between residual metallicity and residual specific inflow rate (blue), residual specific outflow rate (green), residual sSFR (magenta), residual gas fraction (cyan) and residual specific black hole mass (yellow) for central galaxies, at $z<0.1$ (where the residuals are taken w.r.t. the median relations with stellar mass). At low stellar masses $\left(M_{\star} / \mathrm{M}_{\odot}<10^{10.4}\right)$, the gas fraction and specific inflow rate reduce the scatter the most. At high stellar masses $\left(M_{\star} / \mathrm{M}_{\odot}>10^{10.4}\right)$, the specific black hole mass reduces the scatter the most.

ual correlations, as the correlations would have flattened when the fluctuations over these timescales are smoothed.

The relation with the residual specific star formation rate does not change when measured over different time intervals, indicating that for this correlation, short timescales are not the cause. Why the residual correlation does not become steeper like the others is unclear.

We next consider the timescales over which galaxies remain above/below the MZR, independently of the other variables considered. The top panel of Fig. 7 shows the 'memory' of the scatter in the MZR by tracking a sample of galaxies that lie above (or below) the MZR at $z=0$ to higher redshifts. At each cosmic time the fraction of galaxies above (or below) the MZR at that time is determined, until a fraction smaller than 0.2 of the original galaxies remain. The bottom panel of Fig. 7 shows instead the median residual metallicities of these galaxies at the different redshifts.

If the metallicity in the ISM of galaxies had no 'memory', a fraction of 0.5 (grey line, top panel) and a median residual of 0 (grey line, bottom panel) would be expected, as the distribution would be random at $z>0$. Fig. 7 shows clearly that the metallicities of the galaxies do have a memory, as the scenario mentioned above does not hold.

Galaxies above the MZR have on average been above the MZR for $\gtrsim 6 \mathrm{Gyr}$, if their current mass is $M_{\star} / \mathrm{M}_{\odot} \gtrsim 10^{10}$. Galaxies with present-day mass $M_{\star} / \mathrm{M}_{\odot}=10^{9.0 \pm 0.3}$ have been above the MZR for on average $\approx 12 \mathrm{Gyr}$, i.e., their entire lifetime. Galaxies below the MZR have also been below the MZR for on average $\approx 12$ Gyr. This idea is consistent with our hypothesis that processes like star formation, inflows and outflows determine the metallicity, since these also have trends over longer time scales (see figures 1 and 6 . Furthermore, the long timescales involved suggest that the metallicity is driven by the age of the halo formation it- self. When comparing to the work by Matthee \& Schaye (2019) on the memory of the SFR - stellar mass sequence in EAGLE, we see similar timescales $(\approx 10 \mathrm{Gyr})$ appear, which they showed are connected to halo formation. Our results are also broadly speaking consistent with the analysis of the relevant timescales for MZR and star formation rate evolution in the Illustris-TNG simulations, as presented in Torrey et al. (2018), who find that both fluctuate over long timescales.

The asymmetry between above and below the MZR may be due to the fact that galaxies below the MZR have a higher SFR, resulting in a more rapid decrease in stellar mass as we travel back in time, creating a difference between the two samples. Why this difference would result in a longer timescale is not immediately obvious however.

Together, Fig. 6 and Fig. 7] show that the correlations between residual metallicity and residual specific inflow, outflow and star formation rate are consistent with being driven by fluctuations over longer timescales, coinciding with the typical timescales that galaxies have been above the MZR. An extension of our analysis would be to attempt to decompose the fluctuation spectrum explicitly between different timescales, either using Fourier analysis (e.g. Wang \& Lilly 2020b, Iyer et al. 2020) or principal component analysis (Matthee \& Schaye 2019). We leave this as a possible avenue for future work.

\section{SUMMARY}

We have presented our findings concerning the physical origin of the scatter in the Mass-Metallicity Relation (MZR) of central galaxies in the EAGLE simulation and the roles of the gas fraction, specific inflow, outflow and star formation rates. By studying the residual correlations between ISM metallicity and these variables, comparing them, correcting them for one another and studying the timescales involved, it has become clear that although all of these variables are coupled, they also have an independent impact on the scatter in the MZR. The gas fraction, which is most likely an aggregate of the other variables, is the variable correlating best with the scatter in the MZR and the inflow rate seems to play a larger role than the outflow or star formation rates. This section summarises the main results.

(i) There are anti-correlations between the residuals of the MZR, and the residuals of the relations between stellar mass and, respectively, the gas fraction, specific inflow, outflow and star formation rate, at $M_{\star} / \mathrm{M}_{\odot} \lesssim 10^{10}$ (see Fig. 2). These residual correlations are comparable in shape for $M_{\star} / \mathrm{M}_{\odot} \lesssim 10^{10}$. For $M_{\star} / \mathrm{M}_{\odot} \gtrsim 10^{11}$ the shapes deviate compared to lower mass bins: the correlations between residual metallicity and residual sSFR and gas fraction change in sign, showing a positive correlation; the correlations between residual metallicity and residual specific inflow and outflow rate also deviate in shape, showing a positive correlation at negative $\mathrm{x}$-axis residuals, i.e. for relatively low flow rates, and an anti-correlation at positive $\mathrm{x}$-axis residuals, i.e. for relatively high flow rates.

(ii) The residual specific black hole mass (i.e. $\left.\log _{10}\left(M_{\mathrm{BH}}\left(M_{\star}\right) / M_{\star}\right)\right)$ anti-correlates with residual metallicity at $M_{\star} / \mathrm{M}_{\odot} \gtrsim 10^{11}$ and for lower stellar masses also for relatively high black hole masses (see Fig. 3). This suggests that at high black hole masses, AGN feedback strongly influences the gas metallicity of galaxies.

(iii) The relations between the residuals of the MZR and the residual gas fraction, residual specific inflow, outflow and star for- 

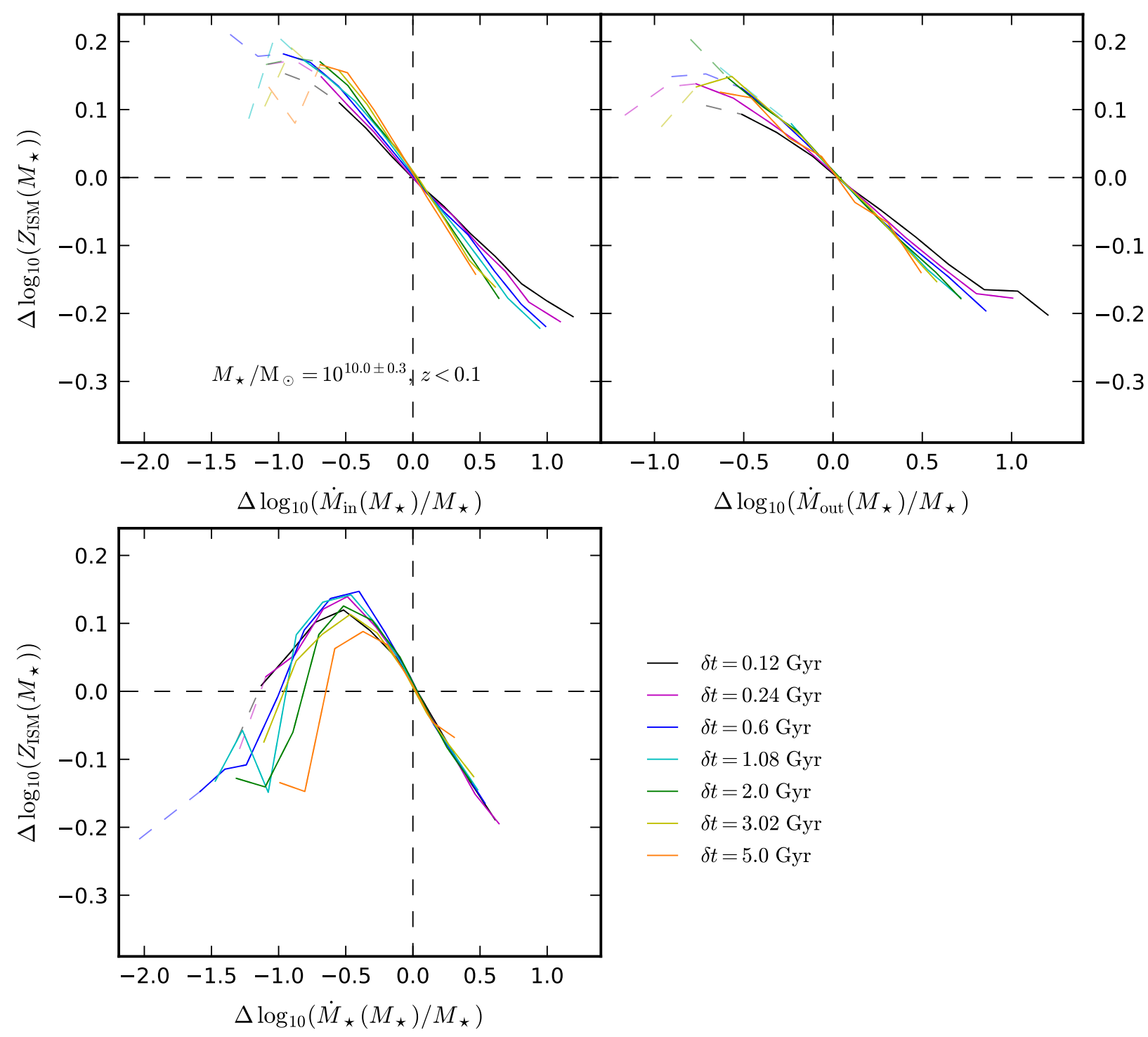

Figure 6. The relations between the median residual of the median MZR and the residuals of the relations with stellar mass of, respectively, specific inflow rate (top left), specific outflow rate (top right) and sSFR (bottom left) for central galaxies with $M_{\star} / \mathrm{M}_{\odot}=10^{10.0 \pm 0.3}$, at $z<0.1$. Different colours correspond to variables averaged over different time intervals. Line styles are as in Fig.2. The specific inflow and outflow rate residual relations steepen when the time interval increases, indicating the importance of longer timescales.

mation rates flatten considerably when corrected for the dependence of metallicity on any of the other variables (see Fig. 4). The relation between residual metallicity and residual specific star formation rate (sSFR) is completely determined by the gas fraction, and almost completely by specific inflow rate and specific outflow rate for non-extreme values $\left(-0.7<\Delta \log _{10}\right.$ (sSFR) $\left.<0.3\right)$. This suggests that all variables are coupled, but that they have some independent influence on the metallicity, of which the influence of the star formation rate is least strong.

(iv) When correcting the scatter in the MZR for the correlations between residual metallicity and residual specific inflow rate, residual specific outflow rate, residual specific star formation rate or the residual gas fraction, and measuring the remaining scatter, the scatter is reduced most (from $\approx 0.13$ dex down to $\approx 0.07$ dex) by the gas fraction, and similarly by the specific inflow rate, at
$M_{\star} / \mathrm{M}_{\odot}<10^{10.2}$ (see Fig. 5). Accounting for the specific outflow or star formation rate reduces the scatter by $\approx 0.025$ dex at $M_{\star} / \mathrm{M}_{\odot}<10^{9.3}$, compared to $\approx 0.04 \mathrm{dex}$ at $M_{\star} / \mathrm{M}_{\odot} \approx 10^{9.6}$. At high stellar masses $\left(M_{\star} / \mathrm{M}_{\odot}>10^{10.2}\right)$ correcting for the specific black hole mass reduces the scatter whereas the contribution of the other variables diminishes.

(v) When averaged over longer time intervals, the anticorrelations of the residual metallicity with the residual specific inflow and outflow rates steepen $\left(M_{\star} / \mathrm{M}_{\odot}=10^{10.0 \pm 0.3}, z<0.1\right)$, indicating that the fluctuations on longer timescales are responsible for the residual correlations (see Fig. 6). The relation with the residual specific star formation rate $\left(M_{\star} / \mathrm{M}_{\odot}=10^{10.0 \pm 0.3}, z<0.1\right)$ does not change when measured over longer time intervals, indicating that fluctuations on short timescales are not responsible for this residual correlation. 


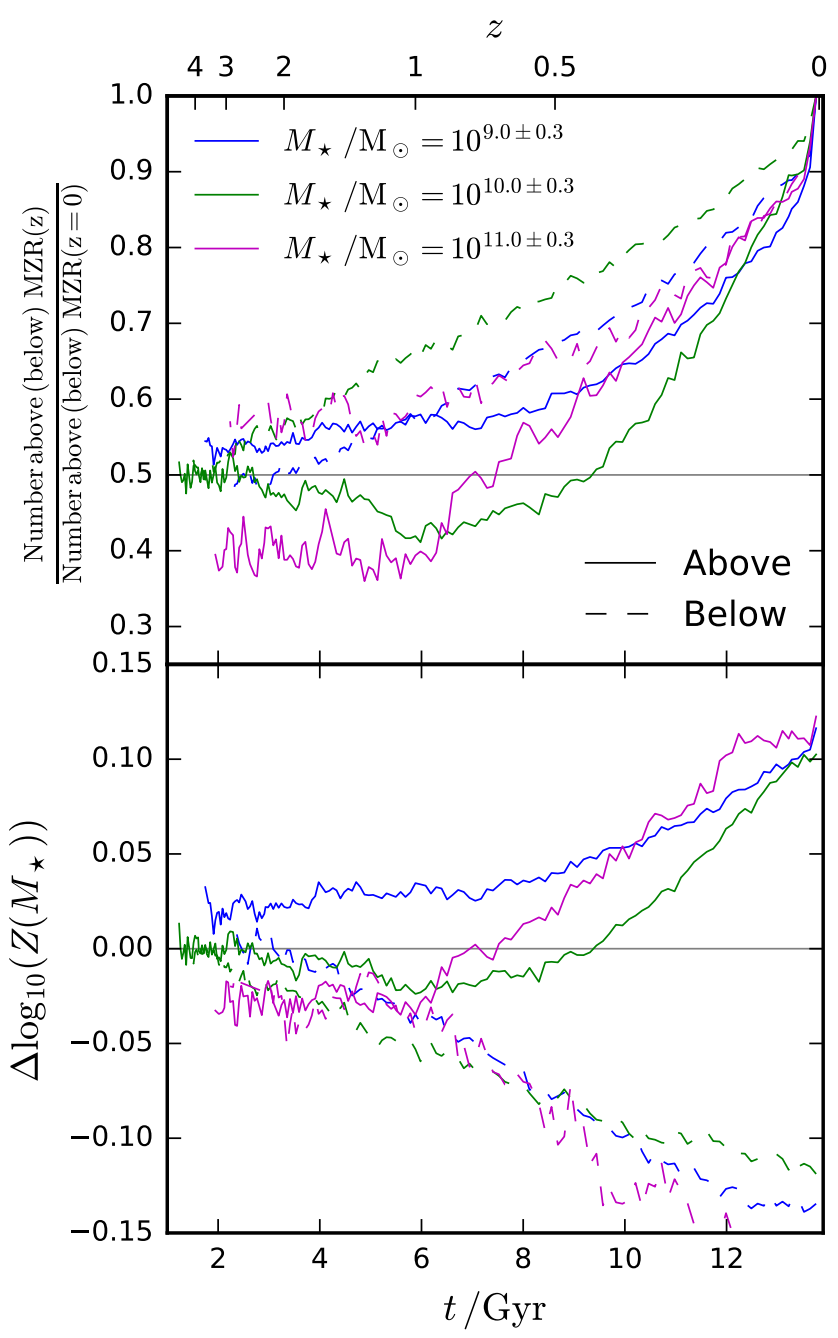

Figure 7. Top: The fraction of galaxies above (solid line style) or below (dashed line style) the MZR as a function of cosmic time, tracking the main progenitors of the sample of galaxies that are above or below the MZR at $z=0(t=13.8 \mathrm{Gyr})$. The grey line at $y=0.5$ indicates what the fraction would be if the distribution were random within the samples at each redshift. Bottom: The median residual metallicity of galaxies in a sample above (solid line style) or below (dashed line style) the MZR at $z=0$ as a function of cosmic times. The grey line at $y=0$ indicates a random median residual at each redshift. In both panels the stellar mass bins (blue: $M_{\star} / \mathrm{M}_{\odot}=10^{9.0 \pm 0.3}$, green: $M_{\star} / \mathrm{M}_{\odot}=10^{10.0 \pm 0.3}$, magenta: $M_{\star} / \mathrm{M}_{\odot}=10^{11.0 \pm 0.3}$ ) refer to masses at $z=0$. The sample above the MZR has been above the MZR for $\gtrsim 6$ Gyr. The sample below the MZR has been below the MZR for $\approx 12 \mathrm{Gyr}$.

(vi) When galaxies with $M_{\star} / \mathrm{M}_{\odot} \gtrsim 10^{10}$ above (below) the MZR at $z=0$ are tracked back in time, these have generally been above (below) the MZR for $\approx 6$ or $\approx 12 \mathrm{Gyr}$, respectively (see Fig. 7). Lower-mass galaxies $\left(M_{\star} / \mathrm{M}_{\odot}=10^{9.0 \pm 0.3}\right)$ have generally been on one side of the MZR for $\approx 12 \mathrm{Gyr}$.

Summarising, star formation, inflows and outflows of gas are coupled processes that all seem to have an independent impact on the metallicity of the ISM, of which the inflows have the most influence. Consistent with the analyis of the Illustris-TNG simulations presented in Torrey et al. (2018), our analysis of the EAGLE simulations indicates that important timescales for these processes are of the order of a few Gyr or longer, which is reflected in how long galaxies stay above/below the MZR, on average. There timescales are also consistent with the recent inference presented in Wang \& Lilly 2020a), based on the residuals measured in observational data.

Overall, we find that the gas fraction is still the variable that correlates best with the metallicity (see also Lagos et al. 2016, De Rossi et al. 2017). We expect that this is primarily because the gas fraction reflects the inflows, outflows and star formation over a range of timescales. This reinforces the general notion that the MZR and its scatter represents an important diagnostic for a range of processes that are critical for galaxy evolution, but which are difficult to observe directly.

\section{ACKNOWLEDGEMENTS}

This work is partly funded by Vici grants 639.043.409 from the Dutch Research Council (NWO).

This work used the DiRAC@Durham facility managed by the Institute for Computational Cosmology on behalf of the STFC DiRAC HPC Facility (www.dirac.ac.uk). The equipment was funded by BEIS capital funding via STFC capital grants ST/K00042X/1, ST/P002293/1, ST/R002371/1 and ST/S002502/1, Durham University and STFC operations grant ST/R000832/1. DiRAC is part of the National e-Infrastructure.

\section{DATA AVAILABILITY}

The EAGLE simulation data are publicly available from http://icc.dur.ac.uk/Eagle/database.php, as described in McAlpine et al. (2016). The processed data underlying this work will be shared on reasonable request to the corresponding author.

\section{REFERENCES}

Almeida J. S., Dalla Vecchia C., 2018, The Astrophysical Journal, 859,109

Bothwell M. S., Maiolino R., Cicone C., Peng Y., Wagg J., 2016, A\&A, 595, A48

Bothwell M. S., Maiolino R., Kennicutt R., Cresci G., Mannucci F., Marconi A., Cicone C., 2013, MNRAS, 433, 1425

Crain R. A. et al., 2015, Monthly Notices of the Royal Astronomical Society, 450, 1937

Curti M., Mannucci F., Cresci G., Maiolino R., 2020, MNRAS, 491, 944

Davé R., Finlator K., Oppenheimer B. D., 2012, MNRAS, 421, 98

Davé R., Thompson R., Hopkins P. F., 2016, MNRAS, 462, 3265

De Lucia G., Xie L., Fontanot F., Hirschmann M., 2020a, MNRAS, 498, 3215

De Lucia G., Xie L., Fontanot F., Hirschmann M., 2020b, MNRAS, 498, 3215

De Rossi M. E., Bower R. G., Font A. S., Schaye J., Theuns T., 2017, Monthly Notices of the Royal Astronomical Society, 472, 3354-3377

Dubois Y., Peirani S., Pichon C., Devriendt J., Gavazzi R., Welker C., Volonteri M., 2016, MNRAS, 463, 3948

Ellison S. L., Patton D. R., Simard L., McConnachie A. W., 2008, ApJ, 672, L107

Finlator K., Davé R., 2008, Monthly Notices of the Royal Astronomical Society, 385, 2181 
Forbes J. C., Krumholz M. R., Burkert A., Dekel A., 2014, MNRAS, 443, 168

Guo Y. et al., 2016, The Astrophysical Journal, 822, 103

Iyer K. G. et al., 2020, MNRAS, 498, 430

Lagos C. d. P. et al., 2016, MNRAS, 459, 2632

Lara-López M. A. et al., 2010, A\&A, 521, L53

Lara-López M. A., De Rossi M. E., Pilyugin L. S., Gallazzi A., Hughes T. M., Zinchenko I. A., 2019, MNRAS, 490, 868

Larson R. B., 1974, MNRAS, 169, 229

Lilly S. J., Carollo C. M., Pipino A., Renzini A., Peng Y., 2013, ApJ, 772, 119

Maiolino R., Mannucci F., 2019, The Astronomy and Astrophysics Review, 27

Mannucci F., Cresci G., Maiolino R., Marconi A., Gnerucci A., 2010, MNRAS, 408, 2115

Matthee J., Schaye J., 2019, Monthly Notices of the Royal Astronomical Society, 484, 915-932

McAlpine S. et al., 2016, Astronomy and Computing, 15, 72

Mitchell P. D., Schaye J., Bower R. G., 2020a, Galactic inflow and wind recycling rates in the eagle simulations

Mitchell P. D., Schaye J., Bower R. G., Crain R. A., 2020b, Monthly Notices of the Royal Astronomical Society, 494, 3971-3997

Mo H. J., Mao S., 2002, MNRAS, 333, 768

Rahmati A., Pawlik A. H., Raičević M., Schaye J., 2013, MNRAS, 430, 2427

Sánchez S. F. et al., 2019, MNRAS, 484, 3042

Sánchez S. F. et al., 2013, A\&A, 554, A58

Schaller M., Dalla Vecchia C., Schaye J., Bower R. G., Theuns T., Crain R. A., Furlong M., McCarthy I. G., 2015, MNRAS, 454, 2277

Schaye J., 2004, The Astrophysical Journal, 609, 667

Schaye J. et al., 2015, MNRAS, 446, 521

Springel V., 2005, MNRAS, 364, 1105

Torrey P. et al., 2018, MNRAS, 477, L16

Torrey P. et al., 2019, MNRAS, 484, 5587

Trayford J. W., Schaye J., 2019, Monthly Notices of the Royal Astronomical Society, 485, 5715

Tremonti C. A. et al., 2004, ApJ, 613, 898

van de Voort F., Schaye J., Booth C. M., Haas M. R., Dalla Vecchia C., 2011, MNRAS, 414, 2458

Vogelsberger M. et al., 2014, MNRAS, 444, 1518

Wang E., Lilly S. J., 2020a, arXiv e-prints, arXiv:2009.01935

Wang E., Lilly S. J., 2020b, ApJ, 895, 25

Yates R. M., Kauffmann G., Guo Q., 2012, MNRAS, 422, 215

Zahid H. J., Dima G. I., Kudritzki R.-P., Kewley L. J., Geller M. J., Hwang H. S., Silverman J. D., Kashino D., 2014, ApJ, 791, 130

Zenocratti L. J., De Rossi M. E., Lara-López M. A., Theuns T., 2020, MNRAS, 496, L33

\section{APPENDIX A: REDSHIFT EVOLUTION}

The redshift evolution varies between the specific inflow rate, specific outflow rate, sSFR and gas fraction residual correlations (figure A1). For the calculations of the specific inflow, outflow and star formation rates different time intervals were used for different redshifts, in approximation of 0.2 times the halo dynamical time, to account for the expansion of the universe. Both the specific inflow rate and gas fraction residual correlations show an increase in steepness of the correlation at higher redshifts for galaxies with $M_{\star} / \mathrm{M}_{\odot}=10^{10.0 \pm 0.3}$. Contrarily, the specific outflow residual correlation shows no clear redshift evolution trend and the sSFR residual correlation seems to flatten at higher redshift although there is no smooth progression. However, overal the redshift evolution is mild. 

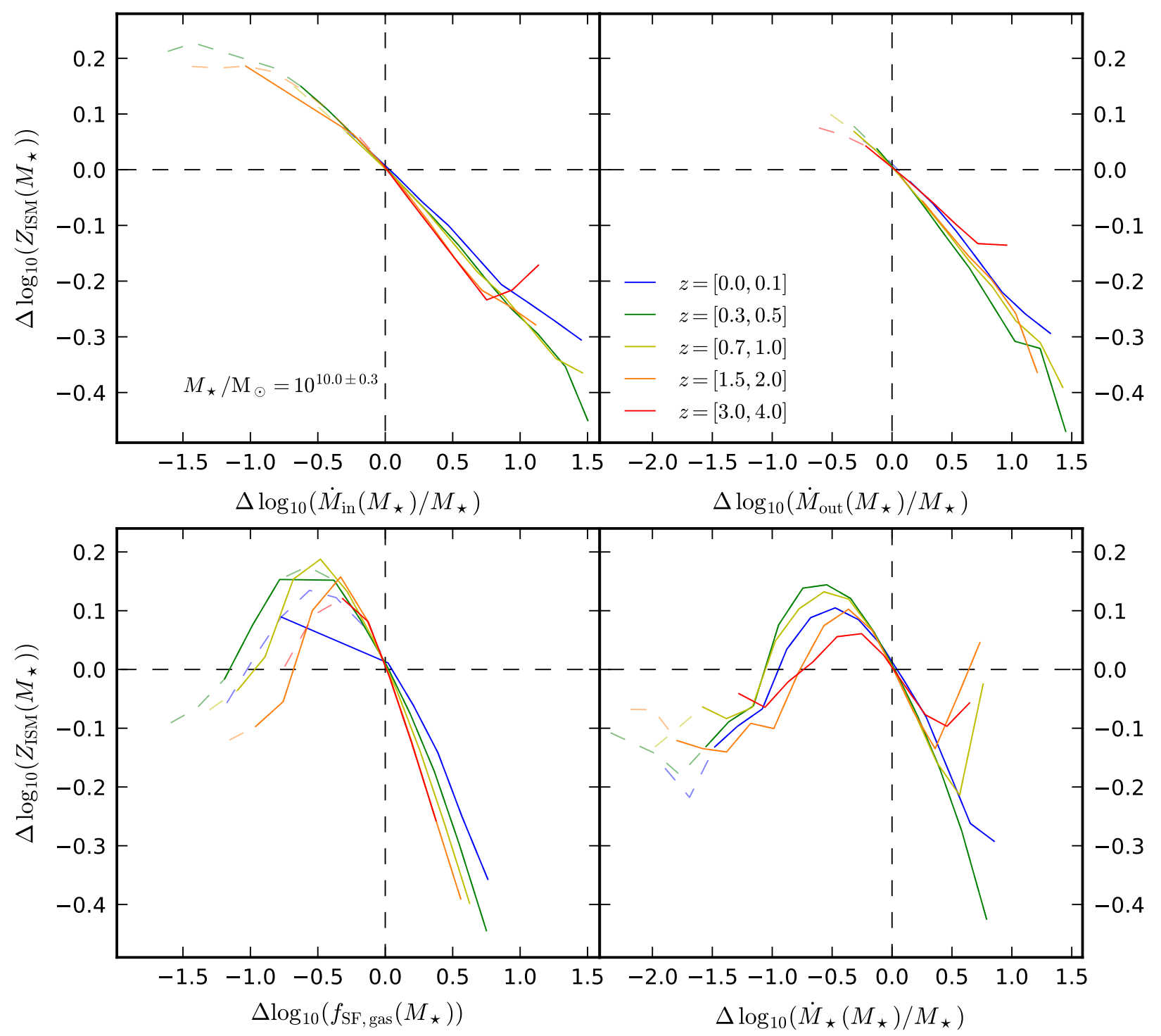

Figure A1. The correlations between residual metallicity and residual specific inflow rate (top left), residual specific outflow rate (top right) residual gas fraction (bottom left) and residual sSFR (bottom right) for galaxies with $M_{\star} / \mathrm{M}_{\odot}=10^{10.0 \pm 0.3}$, at different redshifts. A time interval of 0.24 Gyr for $z<0.1 ; 0.24 \mathrm{Gyr}$ for $z=[0.3,0.5] ; 0.12 \mathrm{Gyr}$ for $z=[0.7,1.0], 0.085 \mathrm{Gyr}$ for $z=[1.5,2.0], 0.043 \mathrm{Gyr}$ for $z=[3.0,4.0]$ between measurements is used, in approximation of 0.2 times the halo dynamical time at these redshifts. Line styles are as in Fig.2. The gas fraction and specific inflow rate residual correlations steepen with redshift while the sSFR residual correlation flattens with redshift. At $z>0.3$ the specific outflow rate residual correlation shows no clear redshift evolution. Overall, the evolution is weak. 